Theorie und Praxis

der Sozialpolitik in der DDR 
Autoren

Dr. Anton Filler

Prof. Dr. sc. Christian Graf

Prof. Dr. sc. Alfred Keck

Prof. Dr. habil. Ottomar Kratsch

Prof. Dr. Herta KuHrig

Prof Dr.-Ing. Ule Lammert

Dr. sc. Gerhard Lippold

Prof. Dr. Kurt Lungwitz

Prof. Dr. sc. Günter Manz

Prof. Dr. Rolf Montag

Dr. sc. Günter Radke

Prof. Dr. Joachim Rittershaus

Dr. sc. Günter Schmunk

Prof. Dr. habil. Gerhard Tietze

Prof. Dr. sc. Gunnar Winkler

Mitautoren an einzelnen Kapiteln

Dr.-Ing. Horst Adami; Jürgen Boje; Peter Brenner; Dr. Johanna Groß;

Dr. habil. Reinhard Gürtler; Dr. Peter Delitz; Dr. Lothar Kühn;

Gerhard Liebscher; Prof. Dr. Ernst Mader; Dr. Hans Mittelbach;

Dr. Rudolf Quapis; Prof. Dr. sc. Joachim Rohde; Dr. Joachim Schindler;

Dr. Christa Seidel, Dr. sc. Renate Walter 


\section{Theorie und Praxis der Sozialpolitik in der DDR}

herausgegeben vom Institut

für Soziologie und Sozialpolitik

der Akademie der Wissenschaften der DDR

durch Günter Manz und Gunnar Winkler

AKADEMIE-VER LAG - BERLIN

1979 
Erschienen im Akademie-Verlag, 108 Berlin, Leipziger Str. 3-4 (c) Akademie-Verlag, Berlin 1979

Lizenz-Nr. $202 \cdot 100 / 36 / 79$

Einband und Schutzumschlag: Peter Schulz

Gesamtherstellung: IV/2/14 VEB Druckerei "Gottfried Wilhelm Leibniz*,

445 Gräfenhainichen $\cdot 5417$

Bestell-Nr. $7536021(6529) \cdot$ LSV 0325

Printed in GDR

DDR 28,- M 